\title{
Jensen type inequalities for twice differentiable functions
}

Abdallah El Frissi, Benharrat Belaïdi*, Zinelaâbidine Lareuch

Department of Mathematics, Laboratory of Pure and Applied Mathematics, University of Mostaganem (UMAB), B. P. 227

Mostaganem-(Algeria)

\author{
Dedicated to George A Anastassiou on the occasion of his sixtieth birthday \\ Communicated by Professor C. Park
}

Abstract
In this paper, we give some Jensen-type inequalities for $\varphi: I \rightarrow \mathbb{R}, I=[\alpha, \beta] \subset \mathbb{R}$, where $\varphi$ is a continuous
function on $I$, twice differentiable on $\stackrel{I}{I}=(\alpha, \beta)$ and there exists $m=\inf _{x \in \dot{I}} \varphi^{\prime \prime}(x)$ or $M=\sup _{x \in I^{\prime \prime}}(x)$.
Furthermore, if $\varphi^{\prime \prime}$ is bounded on $\stackrel{\circ}{I}$, then we give an estimate, from below and from above of Jensen inequalities.@2012 NGA. All rights reserved.

Keywords: Jensen inequality, Convex functions, Twice differentiable functions.

2010 MSC: 26D15.

\section{Introduction and main results}

Throughout this note, we write $I$ and $\stackrel{\circ}{I}$ for the intervals $[\alpha, \beta]$ and $(\alpha, \beta)$ respectively $-\infty \leq \alpha<\beta \leq+\infty$. A function $\varphi$ is said to be convex on $I$ if $\lambda \varphi(x)+(1-\lambda) \varphi(y) \geq \varphi(\lambda x+(1-\lambda) y)$ for all $x, y \in I$ and $0 \leq \lambda \leq 1$. Conversely, if the inequality always holds in the opposite direction, the function is said to be concave on the interval. A function $\varphi$ that is continuous function on $I$ and twice differentiable on $I$ is convex on $I$ if $\varphi^{\prime \prime}(x) \geq 0$ for all $x \in \stackrel{\circ}{I}$ (concave if the inequality is flipped).

The famous inequality of Jensen states that:

\footnotetext{
${ }^{*}$ Corresponding author

Email addresses: elfarissi.abdallah@yahoo.fr (Abdallah El Frissi), belaidi@univ-mosta.dz (Benharrat Belaïdi ), z.latreuch@gmail.com (Zinelaâbidine Lareuch)
} 
Theorem 1.1. ([1], [3]) Let $\varphi$ be a convex function on the interval $I \subset \mathbb{R}, x=\left(x_{1}, x_{2}, \cdots, x_{n}\right) \in I^{n}$ $(n \geq 2)$, let $p_{i} \geq 0, i=1,2, \cdots, n$ and $P_{n}=\sum_{i=1}^{n} p_{i}$. Then

$$
\varphi\left(\frac{1}{P_{n}} \sum_{i=1}^{n} p_{i} x_{i}\right) \leq \frac{1}{P_{n}} \sum_{i=1}^{n} p_{i} \varphi\left(x_{i}\right) .
$$

If $\varphi$ is strictly convex, then inequality in (1.1) is strict except when $x_{1}=x_{2}=\cdots=x_{n}$. If $\varphi$ is a concave function, then inequality in (1.1) is reverse.

Theorem 1.2. [3] Let $\varphi$ be a convex function on $I \subset \mathbb{R}$, and let $f:[0,1] \longrightarrow I$ be a continuous function on $[0,1]$. Then

$$
\varphi\left(\int_{0}^{1} f(x) d x\right) \leq \int_{0}^{1} \varphi(f(x)) d x .
$$

If $\varphi$ is strictly convex, then inequality in (1.2) is strict. If $\varphi$ is a concave function, then inequality in (1.2) is reverse.

In [2], Malamud gave some complements to the Jensen and Chebyshev inequalities and in 4], Saluja gave some necessary and sufficient conditions for three-step iterative sequence with errors for asymptotically quasi-nonexpansive type mapping converging to a fixed point in convex metric spaces. In this paper, we give some inequalities of the above type for $\varphi: I \rightarrow \mathbb{R}$ such that $\varphi$ is a continuous on $I$, twice differentiable on $\stackrel{\circ}{I}$ and there exists $m=\inf _{x \in \dot{I}} \varphi^{\prime \prime}(x)$ or $M=\sup _{x \in I} \varphi^{\prime \prime}(x)$. We obtain the following results:

Theorem 1.3. Let $\varphi: I \longrightarrow \mathbb{R}$ be a continuous function on $I$, twice differentiable on $\stackrel{\circ}{I}, x=\left(x_{1}, x_{2}, \cdots, x_{n}\right) \in$ $I^{n}(n \geq 2)$, let $p_{i} \geq 0, i=1,2, \cdots, n$ and $P_{n}=\sum_{i=1}^{n} p_{i}$.

(i) If there exists $m=\inf _{x \in \dot{I}} \varphi^{\prime \prime}(x)$, then

$$
\begin{aligned}
& \frac{1}{P_{n}} \sum_{i=1}^{n} p_{i} \varphi\left(x_{i}\right)-\varphi\left(\frac{1}{P_{n}} \sum_{i=1}^{n} p_{i} x_{i}\right) \\
\geq & \frac{m}{2}\left(\frac{1}{P_{n}} \sum_{i=1}^{n} p_{i} x_{i}^{2}-\left(\frac{1}{P_{n}} \sum_{i=1}^{n} p_{i} x_{i}\right)^{2}\right) .
\end{aligned}
$$

(ii) If there exists $M=\sup _{x \in I} \varphi^{\prime \prime}(x)$, then

$$
\begin{aligned}
& \frac{1}{P_{n}} \sum_{i=1}^{n} p_{i} \varphi\left(x_{i}\right)-\varphi\left(\frac{1}{P_{n}} \sum_{i=1}^{n} p_{i} x_{i}\right) \\
\leq & \frac{M}{2}\left(\frac{1}{P_{n}} \sum_{i=1}^{n} p_{i} x_{i}^{2}-\left(\frac{1}{P_{n}} \sum_{i=1}^{n} p_{i} x_{i}\right)^{2}\right) .
\end{aligned}
$$

Equality in (1.3) and (1.4) hold if $x_{1}=x_{2}=\cdots=x_{n}$ or if $\varphi(x)=\alpha x^{2}+\beta x+\gamma, \alpha, \beta, \gamma \in \mathbb{R}$.

Theorem 1.4. Let $\varphi: I \longrightarrow \mathbb{R}$ be a continuous function on $I$, twice differentiable on $\stackrel{\circ}{I}$. Suppose that $f:[a, b] \longrightarrow I$ and $p:[a, b] \longrightarrow \mathbb{R}^{+}$are continuous functions on $[a, b]$.

(i) If there exists $m=\inf _{x \in I} \varphi^{\prime \prime}(x)$, then

$$
\frac{\int_{a}^{b} p(x) \varphi(f(x)) d x}{\int_{a}^{b} p(x) d x}-\varphi\left(\frac{\int_{a}^{b} p(x) f(x) d x}{\int_{a}^{b} p(x) d x}\right)
$$




$$
\geq \frac{m}{2}\left(\frac{\int_{a}^{b} p(x)(f(x))^{2} d x}{\int_{a}^{b} p(x) d x}-\left(\frac{\int_{a}^{b} p(x) f(x) d x}{\int_{a}^{b} p(x) d x}\right)^{2}\right) .
$$

(ii) If there exists $M=\sup _{x \in \check{I}} \varphi^{\prime \prime}(x)$, then

$$
\begin{gathered}
\frac{\int_{a}^{b} p(x) \varphi(f(x)) d x}{\int_{a}^{b} p(x) d x}-\varphi\left(\frac{\int_{a}^{b} p(x) f(x) d x}{\int_{a}^{b} p(x) d x}\right) \\
\leq \frac{M}{2}\left(\frac{\int_{a}^{b} p(x)(f(x))^{2} d x}{\int_{a}^{b} p(x) d x}-\left(\frac{\int_{a}^{b} p(x) f(x) d x}{\int_{a}^{b} p(x) d x}\right)^{2}\right) .
\end{gathered}
$$

Equality in (1.5) and (1.6) hold if $\varphi(x)=\alpha x^{2}+\beta x+\gamma, \alpha, \beta, \gamma \in \mathbb{R}$.

Corollary 1.5. Let $\varphi: I \longrightarrow \mathbb{R}$ be a continuous function on $I$, twice differentiable on $\stackrel{\circ}{I}$ and let $f:[a, b] \longrightarrow$ $I$ be a continuous function on $[a, b]$.

(i) If there exists $m=\inf _{x \in I} \varphi^{\prime \prime}(x)$, then

$$
\begin{gathered}
\frac{1}{b-a} \int_{a}^{b} \varphi(f(x)) d x-\varphi\left(\frac{1}{b-a} \int_{a}^{b} f(x) d x\right) \\
\geq \frac{m}{2}\left(\frac{1}{b-a} \int_{a}^{b}(f(x))^{2} d x-\left(\frac{1}{b-a} \int_{a}^{b} f(x) d x\right)^{2}\right) .
\end{gathered}
$$

(ii) If there exists $M=\sup _{x \in I} \varphi^{\prime \prime}(x)$, then

$$
\begin{aligned}
& \frac{1}{b-a} \int_{a}^{b} \varphi(f(x)) d x-\varphi\left(\frac{1}{b-a} \int_{a}^{b} f(x) d x\right) \\
\leq & \frac{M}{2}\left(\frac{1}{b-a} \int_{a}^{b}(f(x))^{2} d x-\left(\frac{1}{b-a} \int_{a}^{b} f(x) d x\right)^{2}\right) .
\end{aligned}
$$

Equality in (1.7) and (1.8) hold if $\varphi(x)=\alpha x^{2}+\beta x+\gamma, \alpha, \beta, \gamma \in \mathbb{R}$.

Corollary 1.6. Let $\varphi: I \longrightarrow \mathbb{R}$ be a continuous function on $I$, twice differentiable on $\stackrel{\circ}{I}, x=\left(x_{1}, x_{2}, \cdots, x_{n}\right) \in$ $I^{n}(n \geq 2)$, let $p_{i} \geq 0, i=1,2, \cdots, n$ and $P_{n}=\sum_{i=1}^{n} p_{i}$. If there exist $m=\inf _{x \in \dot{I}} \varphi^{\prime \prime}(x)$ and $M=\sup _{x \in I} \varphi^{\prime \prime}(x)$, then we have

$$
\begin{aligned}
& \quad \frac{m}{2}\left(\frac{1}{P_{n}} \sum_{i=1}^{n} p_{i} x_{i}^{2}-\left(\frac{1}{P_{n}} \sum_{i=1}^{n} p_{i} x_{i}\right)^{2}\right) \\
& \leq \frac{1}{P_{n}} \sum_{i=1}^{n} p_{i} \varphi\left(x_{i}\right)-\varphi\left(\frac{1}{P_{n}} \sum_{i=1}^{n} p_{i} x_{i}\right) \\
& \leq \frac{M}{2}\left(\frac{1}{P_{n}} \sum_{i=1}^{n} p_{i} x_{i}^{2}-\left(\frac{1}{P_{n}} \sum_{i=1}^{n} p_{i} x_{i}\right)^{2}\right) .
\end{aligned}
$$

Equality in 1.9) occurs, if $\varphi(x)=\alpha x^{2}+\beta x+\gamma, \alpha, \beta, \gamma \in \mathbb{R}$. 
Corollary 1.7. Let $\varphi: I \longrightarrow \mathbb{R}$ be a continuous function on $I$, twice differentiable on $\stackrel{\circ}{I}$, and let $f:[0,1] \longrightarrow$ $I$ be a continuous function on $[0,1]$. If there exist $m=\inf _{x \in \dot{I}} \varphi^{\prime \prime}(x)$ and $M=\sup _{x \in \dot{I}} \varphi^{\prime \prime}(x)$, then we have

$$
\begin{gathered}
\quad \frac{m}{2}\left(\int_{0}^{1}(f(x))^{2} d x-\left(\int_{0}^{1} f(x) d x\right)^{2}\right) \\
\leq \int_{0}^{1} \varphi(f(x)) d x-\varphi\left(\int_{0}^{1} f(x) d x\right) \\
\leq \frac{M}{2}\left(\int_{0}^{1}(f(x))^{2} d x-\left(\int_{0}^{1} f(x) d x\right)^{2}\right) .
\end{gathered}
$$

Equality in (1.10) holds if $\varphi(x)=\alpha x^{2}+\beta x+\gamma \quad \alpha, \beta, \gamma \in \mathbb{R}$.

Corollary 1.8. Let $\varphi: I \longrightarrow \mathbb{R}$ be a convex function on $I$, twice differentiable on $\stackrel{\circ}{I}$, and let $f:[a, b] \longrightarrow I$ be a continuous function on $[a, b]$. If there exists $m=\inf _{x \in \dot{I}} \varphi^{\prime \prime}(x)$, then

$$
\begin{gathered}
\frac{1}{b-a} \int_{a}^{b} \varphi(f(x)) d x-\varphi\left(\frac{1}{b-a} \int_{a}^{b} f(x) d x\right) \\
\geq \frac{m}{2}\left(\frac{1}{b-a} \int_{a}^{b}(f(x))^{2} d x-\left(\frac{1}{b-a} \int_{a}^{b} f(x) d x\right)^{2}\right) \geq 0
\end{gathered}
$$

and

$$
\begin{gathered}
\frac{1}{P_{n}} \sum_{i=1}^{n} p_{i} \varphi\left(x_{i}\right)-\varphi\left(\frac{1}{P_{n}} \sum_{i=1}^{n} p_{i} x_{i}\right) \\
\geq \frac{m}{2}\left(\frac{1}{P_{n}} \sum_{i=1}^{n} p_{i} x_{i}^{2}-\left(\frac{1}{P_{n}} \sum_{i=1}^{n} p_{i} x_{i}\right)^{2}\right) \geq 0 .
\end{gathered}
$$

Corollary 1.9. Let $\varphi: I \longrightarrow \mathbb{R}$ be a concave function on $I$, twice differentiable on $\stackrel{\circ}{I}$, and let $f:[a, b] \longrightarrow I$ be a continuous function on $[a, b]$. If there exists $M=\sup _{x \in \dot{I}} \varphi^{\prime \prime}(x)$, then

$$
\begin{gathered}
\frac{1}{b-a} \int_{a}^{b} \varphi(f(x)) d x-\varphi\left(\frac{1}{b-a} \int_{a}^{b} f(x) d x\right) \\
\leq \frac{M}{2}\left(\frac{1}{b-a} \int_{a}^{b}(f(x))^{2} d x-\left(\frac{1}{b-a} \int_{a}^{b} f(x) d x\right)^{2}\right) \leq 0
\end{gathered}
$$

and

$$
\begin{gathered}
\frac{1}{P_{n}} \sum_{i=1}^{n} p_{i} \varphi\left(x_{i}\right)-\varphi\left(\frac{1}{P_{n}} \sum_{i=1}^{n} p_{i} x_{i}\right) \\
\leq \frac{M}{2}\left(\frac{1}{P_{n}} \sum_{i=1}^{n} p_{i} x_{i}^{2}-\left(\frac{1}{P_{n}} \sum_{i=1}^{n} p_{i} x_{i}\right)^{2}\right) \leq 0 .
\end{gathered}
$$

Remark 1.10. In the above if $\varphi \in C^{2}([\alpha, \beta])$, then we can replace inf and sup by min and max respectively. 


\section{Lemma}

Our proofs depend mainly upon the following lemma.

Lemma 2.1. Let $\varphi$ be a convex function on $I \subset \mathbb{R}$ and differentiable on $\stackrel{\circ}{I}$. Suppose that $f:[a, b] \longrightarrow I$ and $p:[a, b] \longrightarrow \mathbb{R}^{+}$are continuous functions on $[a, b]$. Then

$$
\varphi\left(\frac{\int_{a}^{b} p(x) f(x) d x}{\int_{a}^{b} p(x) d x}\right) \leq \frac{\int_{a}^{b} p(x) \varphi(f(x)) d x}{\int_{a}^{b} p(x) d x} .
$$

If $\varphi$ is strictly convex, then inequality in 2.1) is strict. If $\varphi$ is a concave function, then inequality in 2.1) is reverse.

Proof. Suppose that $\varphi$ is a convex function on $I \subset \mathbb{R}$ and differentiable on $\stackrel{\circ}{I}$. Then for each $x, y \in \stackrel{\circ}{I}$, we have

$$
\varphi(x)-\varphi(y) \geq(x-y) \varphi^{\prime}(y) .
$$

Replace $x$ by $f(x)$ and set $y=\frac{\int_{a}^{b} p(x) f(x) d x}{\int_{a}^{b} p(x) d x}$ in 2.2 , we obtain

$$
\begin{gathered}
\varphi(f(x))-\varphi\left(\frac{\int_{a}^{b} p(x) f(x) d x}{\int_{a}^{b} p(x) d x}\right) \\
\geq\left(f(x)-\frac{\int_{a}^{b} p(x) f(x) d x}{\int_{a}^{b} p(x) d x}\right) \varphi^{\prime}\left(\frac{\int_{a}^{b} p(x) f(x) d x}{\int_{a}^{b} p(x) d x}\right) .
\end{gathered}
$$

Multiplying both sides of inequality $(2.3)$ by $p(x)$ we obtain

$$
\begin{gathered}
p(x) \varphi(f(x))-p(x) \varphi\left(\frac{\int_{a}^{b} p(x) f(x) d x}{\int_{a}^{b} p(x) d x}\right) \\
\geq\left(p(x) f(x)-p(x) \frac{\int_{a}^{b} p(x) f(x) d x}{\int_{a}^{b} p(x) d x}\right) \varphi^{\prime}\left(\frac{\int_{a}^{b} p(x) f(x) d x}{\int_{a}^{b} p(x) d x}\right) .
\end{gathered}
$$

By integration in (2.4) we obtain (2.1).

\section{Proof of the Theorems}

Proof of Theorem 1.3. Suppose that $\varphi: I \rightarrow \mathbb{R}$ is a continuous function on $I$ and twice differentiable on $\stackrel{\circ}{I}$. Set $g(x)=\varphi(x)-\frac{m}{2} x^{2}$. Differentiating twice times both sides of $g$ we get $g^{\prime \prime}(x)=\varphi^{\prime \prime}(x)-m \geq 0$. Then $g$ is a convex function on $I$. By formula (1.1), we have

$$
g\left(\frac{1}{P_{n}} \sum_{i=1}^{n} p_{i} x_{i}\right) \leq \frac{1}{P_{n}} \sum_{i=1}^{n} p_{i} g\left(x_{i}\right)
$$

which implies that

$$
\begin{gathered}
\varphi\left(\frac{1}{P_{n}} \sum_{i=1}^{n} p_{i} x_{i}\right)-\frac{m}{2}\left(\frac{1}{P_{n}} \sum_{i=1}^{n} p_{i} x_{i}\right)^{2} \\
\leq \frac{1}{P_{n}} \sum_{i=1}^{n} p_{i} \varphi\left(x_{i}\right)-\frac{m}{2} \frac{1}{P_{n}} \sum_{i=1}^{n} p_{i} x_{i}^{2}
\end{gathered}
$$


Then, by $(3.2)$ we can write

$$
\begin{aligned}
& \frac{1}{P_{n}} \sum_{i=1}^{n} p_{i} \varphi\left(x_{i}\right)-\varphi\left(\frac{1}{P_{n}} \sum_{i=1}^{n} p_{i} x_{i}\right) \\
\geq & \frac{m}{2}\left(\frac{1}{P_{n}} \sum_{i=1}^{n} p_{i} x_{i}^{2}-\left(\frac{1}{P_{n}} \sum_{i=1}^{n} p_{i} x_{i}\right)^{2}\right) .
\end{aligned}
$$

If we put $g(x)=-\varphi(x)+\frac{M}{2} x^{2}$, then by differentiating both sides of $g$ we get $g^{\prime \prime}(x)=-\varphi^{\prime \prime}(x)+M \geq 0$. Hence $g$ is a convex function on $I$ and by similar proof as above, we obtain

$$
\begin{aligned}
& \frac{1}{P_{n}} \sum_{i=1}^{n} p_{i} \varphi\left(x_{i}\right)-\varphi\left(\frac{1}{P_{n}} \sum_{i=1}^{n} p_{i} x_{i}\right) \\
\leq & \frac{M}{2}\left(\frac{1}{P_{n}} \sum_{i=1}^{n} p_{i} x_{i}^{2}-\left(\frac{1}{P_{n}} \sum_{i=1}^{n} p_{i} x_{i}\right)^{2}\right) .
\end{aligned}
$$

Proof of Theorem 1.4. Suppose that $\varphi: I \rightarrow \mathbb{R}$ is a continuous function on $I$ and twice differentiable on $\stackrel{\circ}{I}$. Set $g(x)=\varphi(x)-\frac{m}{2} x^{2}$. Differentiating both sides of $g$ we get $g^{\prime \prime}(x)=\varphi^{\prime \prime}(x)-m \geq 0$. Hence $g$ is a convex function on $I$ and by formula (2.1) we have

$$
g\left(\frac{\int_{a}^{b} p(x) f(x) d x}{\int_{a}^{b} p(x) d x}\right) \leq \frac{\int_{a}^{b} p(x) g(f(x)) d x}{\int_{a}^{b} p(x) d x}
$$

which implies that

$$
\begin{aligned}
& \varphi\left(\frac{\int_{a}^{b} p(x) f(x) d x}{\int_{a}^{b} p(x) d x}\right)-\frac{m}{2}\left(\frac{\int_{a}^{b} p(x) f(x) d x}{\int_{a}^{b} p(x) d x}\right)^{2} \\
& \leq \frac{\int_{a}^{b} p(x) \varphi(f(x)) d x}{\int_{a}^{b} p(x) d x}-\frac{m}{2} \frac{\int_{a}^{b} p(x)(f(x))^{2} d x}{\int_{a}^{b} p(x) d x} .
\end{aligned}
$$

Then by (3.6), we can write

$$
\begin{gathered}
\frac{\int_{a}^{b} p(x) \varphi(f(x)) d x}{\int_{a}^{b} p(x) d x}-\varphi\left(\frac{\int_{a}^{b} p(x) f(x) d x}{\int_{a}^{b} p(x) d x}\right) \\
\geq \frac{m}{2}\left(\frac{\int_{a}^{b} p(x)(f(x))^{2} d x}{\int_{a}^{b} p(x) d x}-\left(\frac{\int_{a}^{b} p(x) f(x) d x}{\int_{a}^{b} p(x) d x}\right)^{2}\right) .
\end{gathered}
$$

If we put $g(x)=\varphi(x)-\frac{M}{2} x^{2}$, then by differentiating both sides of $g$, we get $g^{\prime \prime}(x)=\varphi^{\prime \prime}(x)-M \leq 0$. Thus, $g$ is a concave function on $I$ and by a similar proof as above, we obtain

$$
\begin{gathered}
\frac{\int_{a}^{b} p(x) \varphi(f(x)) d x}{\int_{a}^{b} p(x) d x}-\varphi\left(\frac{\int_{a}^{b} p(x) f(x) d x}{\int_{a}^{b} p(x) d x}\right) \\
\leq \frac{M}{2}\left(\frac{\int_{a}^{b} p(x)(f(x))^{2} d x}{\int_{a}^{b} p(x) d x}-\left(\frac{\int_{a}^{b} p(x) f(x) d x}{\int_{a}^{b} p(x) d x}\right)^{2}\right) .
\end{gathered}
$$




\section{References}

[1] I. Budimir, S. S. Dragomir and J. Pečarić, Further reverse results for Jensen's discrete inequality and applications in information theory, J. Inequal. Pure Appl. Math. 2 (2001), no. 1, Article 5, 1-14. 1.1

[2] S. M. Malamud, Some complements to the Jensen and Chebyshev inequalities and a problem of W. Walter, Proc. Amer. Math. Soc. 129 (2001), no. 9, 2671-2678. 1

[3] D. S. Mitrinović, J. E. Pečarić and A. M. Fink, Classical and new inequalities in analysis. Mathematics and its Applications (East European Series), 61. Kluwer Academic Publishers Group, Dordrecht, 1993. 1.1, 1.2

[4] G. S. Saluja, Convergence of fixed point of asymptoticaly quasi-nonexpansive type mappings in convex metric spaces, J. Nonlinear Sci. Appl. 1 (2008), no. 3, 132-144. 1 